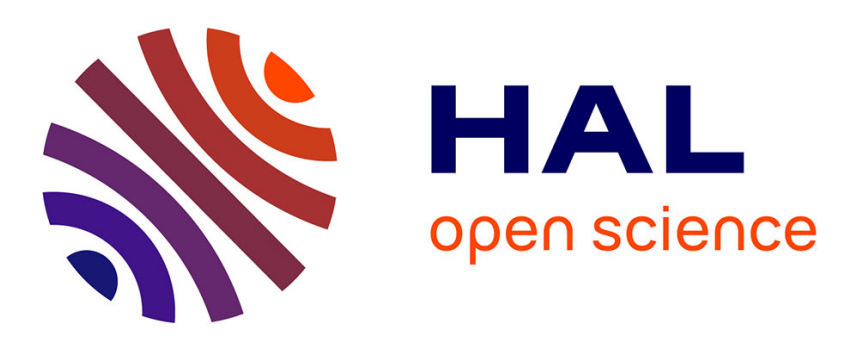

\title{
Guaranteed cost control design for descriptor systems with time-varying delays
}

Mohamad Taki Asghar, Marc Jungers, Irinel-Constantin Morarescu, Ahmed Khelassi, Julien Francken

\section{- To cite this version:}

Mohamad Taki Asghar, Marc Jungers, Irinel-Constantin Morarescu, Ahmed Khelassi, Julien Francken. Guaranteed cost control design for descriptor systems with time-varying delays. 56th IEEE Conference on Decision and Control, CDC 2017, Dec 2017, Melbourne, Australia. hal-01653188

\section{HAL Id: hal-01653188 \\ https://hal.science/hal-01653188}

Submitted on 1 Dec 2017

HAL is a multi-disciplinary open access archive for the deposit and dissemination of scientific research documents, whether they are published or not. The documents may come from teaching and research institutions in France or abroad, or from public or private research centers.
L'archive ouverte pluridisciplinaire HAL, est destinée au dépôt et à la diffusion de documents scientifiques de niveau recherche, publiés ou non, émanant des établissements d'enseignement et de recherche français ou étrangers, des laboratoires publics ou privés. 


\title{
Guaranteed cost control design for descriptor systems with time-varying delays
}

\author{
Mohamad Taki Asghar, Marc Jungers, Irinel-Constantin Morărescu, Ahmed Khelassi and Julien Francken
}

\begin{abstract}
Motivated by a real problem in cold rolling of steel, we propose a design methodology of guaranteed cost controllers for descriptor type systems in presence of multiple time-varying delays. We first analyse the existence and uniqueness of solutions for the class of systems under study. This enable us to define the compatible initial conditions for this class of systems and show that for the closed-loop dynamics they depend on the controller. Consequently, we provide a methodology to avoid this dependency. Secondly, we consider the problem of designing a controller that stabilizes the system and ensures some performance guarantees. The proposed solution consists of minimizing a cost function related to the energetic aspects of the system. The main tool used for the control design is a modified Lyapunov-Krasovskii functional that takes into account the singularity of the system. Our solution can be easily implemented since the controller is obtained by solving some linear matrix inequalities (LMIs). A numerical example illustrates the implementation of our results.
\end{abstract}

\section{INTRODUCTION}

Stability analysis and control design for dynamical systems in presence of time-delays is a problem of recurring interests [1], [2]. Although there exists an extensive literature on the analysis of systems affected by fixed point-wise delays, we can also find many results related to dynamics affected by distributed [3], [4] and time-varying delays [5], [6]. Despite this rich literature, the research on time-delay systems is still very active and new computationally oriented stability criteria [7], [8] are regularly proposed. This is certainly due to the demand of industrial companies for easy and fast implementable analysis and control design tools.

Beside the delay presence, another features that characterizes many real processes are the complexity and the heterogeneity of the dynamics describing them. Often the evolution of the state variables of the system is constrained by some algebraic relations with an explicit physical meaning. This complex behavior is mathematically captured by the class of descriptor or singular systems [9], [10].

The analysis of the class of descriptor systems with tine-varying delays discussed in this paper is motivated by an industrial application. One important process in metallurgy is

M. T. Asghar, M. Jungers and I.-C. Morărescu are with Université de Lorraine, CRAN, UMR 7039, 2 Avenue de la Forêt de Haye, Vandœuvre-lès-Nancy, France and CNRS, CRAN, UMR 7039. Corresponding author marc.jungers@univ-lorraine.fr.

M. T. Asghar, Ahmed Khelassi and Julien Francken are with Control and Measurement Department, ArcelorMittal Maizières R\&D, Voie Romaine BP 30320, F-57283 Maizières-lès-Metz, France.

This work has been supported by a grant of the French Agency for Research and Technology (ANRT). the metal rolling in which the metal strip achieves a desired thickness. Tandem Cold Mill (TCM) [11] is composed by several stands that sequentially reduce the thickness of the metal strip. Therefore, the thickness of the metal sheet entering one stand is the delayed version of the thickness of the sheet that get out from the precedent stand. In other words, the delays consist in the transportation durations of the metal strip between two consecutive stands. The speed of the metal strip varies in function of the thickness reduction, accordingly to the flow conservation law, which is the algebraic relation stating that the flow of metal entering one stand is equal to the flow of metal exiting the stand. Consequently, the time-delay introduced by the transport from one stand to the next is also time-varying. In [12], we showed that the behavior of the TCM, with five stands, is accurately described by a non-linear descriptor system with four time-varying delays. We also proposed a linearization of this model around the operating point and we proved that the linearized model is valid in a wide domain of parameters variation.

Up to now, several models have been elaborated [11], [13]-[16], to accurately express the challenges of this system. These models have been studied and examined with respect to industrial data in order to calibrate their coefficients and adapt them to the existing mills. They have been used for control synthesis, system diagnosis, signal measurements, estimation and observations, quantitative and qualitative studies [17]. While a lot of efforts have been oriented toward model accuracy and calibration the control design remains quite basic since nowadays the rolling is controlled by means of PIDs controllers. Due to the evolution of the technology, the specifications required for the steel sheets continuously change asking for thinner sheets with an increased performance assessment (mainly hardness, but also flatness...) [18]. In order to accomplish this objective one needs to adopt new control strategies aiming at: reaching the required specifications of the steel sheets; taking into account the complex interactions inside the system; reducing the energy cost of the rolling process and finally being ready for the further introduction of new actuators such as flexible lubrication [19], [20].

The main contribution of this work is related to the control design with performance guarantees for descriptor systems in presence of time-varying delays. We emphasize that stability analysis and the control design for descriptor systems with fixed delays has been considered in [21]. When the delays are time-varying the results are oriented more toward stability analysis [22]-[24]. To the best of the author knowledge, 
the control design with performance guarantees was not yet addressed for this class of systems. We are treating this problem motivated by the ArcelorMittal application, related to the TCM.

The rest of the paper is organized as follows. Section II introduces the time-varying delays descriptor system under study and formulates the state-feedback control design problem that will be solved. The existence and uniqueness of trajectory of the underlined system are provided in section III. The main results on the control design are gathered in section IV: firstly conditions ensuring the stabilization of the time-varying descriptor system are proposed as linear matrix inequalities and secondly an optimization problem, that minimizes an upper bound of the performance cost is provided. We numerically illustrate our results in section $\mathrm{V}$ and we give some concluding remarks in section VI.

\section{Notation}

In the rest of the paper, the following notations will be used. $\mathbb{R}$ is the set of real numbers. For all $\theta \in[-h, 0]$ we us the notation $x_{t}(\theta)=x(t+\theta) .|x|$ is the absolute value of $x \in \mathbb{R} .\|x\|$ is the Euclidean norm of $x \in \mathbb{R}^{n} .\|A\|$ denotes the Euclidean induced norm of the matrix $A \in \mathbb{R}^{n \times n} . \lambda(A)$ denotes the vector of the eigenvalues of the matrix $A$ and $A_{(i)}$ its $i$ th row. $I_{n} \in \mathbb{R}^{n \times n}$ represents the identity matrix of dimension $n$. The relation $P>0$ refers to that the matrix $P \in \mathbb{R}^{n \times n}$ is positive definite. The superscript $T$ means the transpose, and the symbol $\star$ represents the term induced by symmetry. $\operatorname{diag}\{\ldots\}$ stands for a block diagonal matrix. $\mathrm{He}(\cdot)$ is the matrix Hermitian operator. Finally, $\operatorname{Tr}(\cdot)$ is the trace of a square matrix and $\mathbb{E}(\cdot)$ is the expectation of stochastic variables.

\section{STUdied SYSTEM AND ISSUE TO SOlVE}

Motivated by the preliminary modeling of the TCM in [12] with $\ell+1$ stands, in the sequel we consider the following singular system with $\ell$-time-varying delays:

$$
\begin{aligned}
E \dot{x}(t) & =A_{0} x(t)+\sum_{i=1}^{\ell} A_{i} x\left(t-\tau_{i}(t)\right)+B u(t) \\
& =f\left(x(t), x_{t}\left(-\tau_{1}(t)\right), \ldots, x_{t}\left(-\tau_{\ell}(t)\right), u(t)\right)
\end{aligned}
$$

where $x(t) \in \mathbb{R}^{n}$ and $u(t) \in \mathbb{R}^{m}$ are the state and input vectors respectively. $E, A_{i} \in \mathbb{R}^{n \times n}$ and $B \in \mathbb{R}^{n \times m}$ are constant matrices. The matrix $E$ is singular and such that $\operatorname{rank}(E)=r<n$. For the TCM, the state gathers physical variables and naturally the system is a concatenation of $r$ nonlinear differential equations and $(n-r)$ nonlinear algebraic equations leading to a singular matrix $E$ of type $E=\operatorname{diag}\left(I_{r}, 0_{n-r}\right)$. We will assume this value in the sequel of the paper. We introduce the associated partition of matrices $A_{i}, i \in\{0,1, \ldots, \ell\}$ as follows into two subspaces of dimensions $r$ and $(n-r)$

$$
A_{i}=\left[\begin{array}{c:c}
A_{i 1} & A_{i 2} \\
\hdashline A_{i 3} & A_{i 4}
\end{array}\right], \forall i \in\{0, \ldots, \ell\}, B=\left[\begin{array}{c}
B_{1} \\
\hdashline B_{2}
\end{array}\right] .
$$

The same decomposition holds for the notation $x(t)=$ $\left(\begin{array}{l}x_{1}(t) \\ x_{2}(t)\end{array}\right)$ and $u(t)=\left(\begin{array}{l}u_{1}(t) \\ u_{2}(t)\end{array}\right)$.

The delays $\tau_{1}(\cdot), \ldots, \tau_{\ell}(\cdot)$ in the dynamics (1) consist in propagation times of the metal strip between the stands: they are state-dependent (mainly strip speed dependent) but we will consider here only robustness with respect to these delays and assume only they are time-dependent with the following assumptions:

- The delays are causal and upper bounded

$$
0 \leq \tau_{i}(t) \leq h_{i}, h_{i}>0, \forall i \in\{1, \ldots, \ell\} .
$$

We denote $\bar{h}=\max \left\{h_{i} \mid i=1, \ldots, \ell\right\}$.

- The delays are derivable functions of time $t$ and

$$
\dot{\tau}_{i}(t) \leq d_{i}<1, \forall i \in\{1, \ldots, \ell\} .
$$

It should be pointed out that the conditions (4) and (5) are physically natural for TCM, due mainly to the bounded speed and also the bounded acceleration of the metal strip. In inequalities (5), the upper bound $d_{i}$ are strictly less than 1 , thanks to the fact that the strip does not fold and that the passing order of the slices of the strip into the stands is preserved.

To complete the definition of our system we have to introduce the initial condition as:

$$
x(\theta)=\phi(\theta), \quad \forall \theta \in\left[t_{0}-\bar{h}, t_{0}\right]
$$

with $\phi \in \mathcal{C}_{\bar{h}, n}=\mathcal{C}\left(\left[t_{0}-\bar{h}, t_{0}\right], \mathbb{R}^{n}\right)$. The notation $\phi(t)=\left(\begin{array}{l}\phi_{1}(t) \\ \phi_{2}(t)\end{array}\right)$ is induced by the decomposition of $E$. Additional conditions allowing $\phi(\cdot)$ to be compatible, that is ensuring the existence and the uniqueness of a continuous trajectory issued from (1) and (6), will be given in the following section.

Selecting a control law is guided, in practice, by the willingness to reach a collection of specifications related to the TCM and to the product. A large number of specifications can be formulated into a quadratic cost function to minimize. The induced multi-objective optimization problem may be recasted into a mono-objective one by considering a suitable weighting sum of these specifications defined as

$$
\mathcal{J}(\phi, u)=\int_{t_{0}}^{+\infty}\left(x^{T}(s) Q x(s)+u^{T}(s) R u(s)\right) \mathrm{d} s,
$$

where $Q=Q^{T} \geq 0_{n}$ and $R=R^{T}>0_{m}$.

The tuning parameters are thus these weighting coefficients. Furthermore if the trajectory related to the initial condition $\phi(\cdot)$ and the control $u(\cdot)$ exists and is unique, the cost $\mathcal{J}$ depends only on $\phi(\cdot)$ and $u(\cdot)$.

Remark 1: It should be noticed that the criterion $\mathcal{J}$ defined by (7) does not contain time-delayed quadratic terms. This choice is for simplicity, an easy physical interpretation of each term as well as to avoid redundancy of variables over the integral support $\left[t_{0},+\infty\right)$. Nevertheless, quadratic terms with respect to delayed states $x_{s}\left(\tau_{i}(s)\right),(i \in\{1, \ldots, \ell\})$ may be added thanks to the use of the extended state $\xi(t)$ given by (22). 
Beside the issue of existence and uniqueness of a continuous trajectory, the problem that is investigated in this paper is defined as follows

Problem 1: Considering the system (1) and (6) under the assumptions (4) and (5), design a control law $u(\cdot)$ stabilizing the system and in addition minimizing the quadratic cost function $\mathcal{J}$ given by Equation (7).

The tools of optimal control theory are not well adapted to the presence of time-delays [2]. The main idea to solve Problem 1 is to restrict the control synthesis to a particular class of controllers, here the class of state-feedbacks

$$
u(t)=K x(t)
$$

and to use the approach of Lyapunov-Krasovskii functionals firstly ensuring the stabilization of the system and secondly providing an upper bound of the cost function $\mathcal{J}$.

\section{EXISTENCE AND UNIQUENESS OF SOLUTION}

The existence (but also the uniqueness) of a solution of the system (1) and (6) is not always guaranteed mainly because of the presence of the singular matrix $E$. Therefore, in this section we determine sufficient conditions on the initial condition $\phi(\cdot)$ ensuring the existence and the uniqueness of a continuous trajectory of the system (1) and (6).

Before presenting the result of this section, let us recall some existing contributions in the literature on the existence and uniqueness of solution for descriptor systems. According to [9], [10], [25], the regularity and free impulsiveness of the delay-free singular system (i.e. $A_{1}=\ldots=A_{\ell}=0$ ) ensure the existence and uniqueness of its solution. In the case of singular systems with constant delays, as mentioned in [22], [25], the regularity and the free impulsiveness accompanied by a compatible continuous initial function $\phi(\cdot)$ ensure the existence and the uniqueness of the solution. The regularity and free impulsiveness are required to be able to locally propagate the definition of a continuous trajectory. The compatible initial condition is required to avoid jumps at time $t=t_{0}$. This issue is known as the Initial Value Problem (IVP). Inappropriate initial conditions can induce jumps [26] which may propagate through the solution due to delays. This may affect the uniqueness of the solution.

A classical way of proving the existence and uniqueness of continuous solution for singular systems with constant delays is to reformulate the dynamics as a neutral delay system with constant coefficients [22], [25], [27]. However, it is not trivial to extend this approach to the case of descriptor systems with time-varying delays. The main difficulty is related to the fact that, in this case, the delay derivative is not zero and supplementary terms appear in the neutral dynamics formulation.

Theorem 1: Consider a regular impulse free singular system with time-varying delays described by (1) and (6). The system admits a unique continuous solution $x(t, \phi)$ over $\left[t_{0}-\bar{h} ; \infty\right)$ if the time delays $\tau_{i}(t), i=1, \ldots, \ell$ are continuous and the initial value problem IVP $\phi(\cdot)$ verifies the following condition:

$$
\begin{aligned}
0=A_{03} & \phi_{1}\left(t_{0}\right)+A_{04} \phi_{2}\left(t_{0}\right) \\
& +\sum_{j=1}^{2} \sum_{i=1}^{\ell} A_{i, j+2} \phi_{j}\left(t_{0}-\tau_{i}\left(t_{0}\right)\right)+B_{2} u\left(t_{0}\right) .
\end{aligned}
$$

Proof: The proof is inspired by the one in [9], that is related to the constant delays and contains two parts. The first one ensures that the algebraic relation in system (1), that is their $(n-r)$ last lines, is verified at $t=t_{0}$. The second one allows to propagate the existence and continuity of the trajectory.

The first part concerns only the algebraic relation at time $t=t_{0}$. Thanks to the decomposition of $E$, we should have the algebraic relation concerning the state as follows, $\forall t \geq$ $t_{0}$,

$$
\begin{aligned}
0=A_{03} x_{1}(t) & +A_{04} x_{2}(t) \\
& +\sum_{j=1}^{2} \sum_{i=1}^{\ell} A_{i, j+2} x_{j}\left(t-\tau_{i}(t)\right)+B_{2} u(t) .
\end{aligned}
$$

At time $t=t_{0}$, by substituting $x(\cdot)$ by $\phi(\cdot)$, thanks to Equation (6), we obtain the desired relation (9).

The second part is proven based on the structure of the dynamics (1), on the regularity of the time delays and on the regularity of the initial condition $\phi(\cdot)$. Precisely, using the Cauchy-Schwartz inequality one can write the following:

$$
\begin{aligned}
& \| f\left(x\left(t_{1}\right), x_{t_{1}}\left(-\tau_{1}\left(t_{1}\right)\right), \ldots, x_{t_{1}}\left(-\tau_{\ell}\left(t_{1}\right)\right), u\left(t_{1}\right)\right) \\
& -f\left(x\left(t_{2}\right), x_{t_{2}}\left(-\tau_{1}\left(t_{2}\right)\right), \ldots, x_{t_{2}}\left(-\tau_{\ell}\left(t_{2}\right)\right), u\left(t_{2}\right)\right) \| \\
& \leq\left\|\left[\begin{array}{lllll}
A_{0} & A_{1} & \ldots & A_{d} & B
\end{array}\right]\right\| \times \\
& \left\|\left[\begin{array}{c}
x\left(t_{1}\right)-x\left(t_{2}\right) \\
x\left(t_{1}-\tau_{1}\left(t_{1}\right)\right)-x\left(t_{2}-\tau_{1}\left(t_{2}\right)\right) \\
\cdots \\
x\left(t_{1}-\tau_{\ell}\left(t_{1}\right)\right)-x\left(t_{2}-\tau_{\ell}\left(t_{2}\right)\right) \\
u\left(t_{1}\right)-u\left(t_{2}\right)
\end{array}\right]\right\| .
\end{aligned}
$$

This yields that the function $f(\cdot, \ldots, \cdot, \cdot)$ is globally Lipschitz if the time delays, the control input and the past of the trajectory (that is at least $\phi(\cdot)$ ) are continuous time functions. Consequently, assuming that a unique trajectory exists and is continuous until time $t$, one can uniquely define locally, by propagation, the value of the continuous trajectory $x_{1}(s)$ over $s \in[t, t+\delta t]$, with $\delta t$ small enough, using the expression of $\dot{x}_{1}(t)$. The regularity of the system implies that $A_{04}$ is not singular. That permits to define the continuous value of $x_{2}(s)$ at each instant $s \in[t, t+\delta t]$ by knowing $x_{1}(s)$ over $s \in[t, t+\delta t]$.

Remark 2: It should be noticed that the relation (9) introduces a constraint between the initial condition $\phi(\cdot)$ and the control input $u(\cdot)$. As a consequence, if the initial condition is imposed, the control synthesis should take it into account. On another side, if the control law is given, then its properties will be guaranteed only for the set of functions $\phi \in \mathcal{C}_{\bar{h}, n}$ verifying the induced relation (9). 
Remark 3: When the state feedback control law (8) is applied to the system (1), the condition (9) may be rewritten as

$$
\begin{array}{r}
0=\left(A_{03}+B_{2} K_{1}\right) \phi_{1}\left(t_{0}\right)+\left(A_{04}+B_{2} K_{2}\right) \phi_{2}\left(t_{0}\right) \\
+\sum_{j=1}^{2} \sum_{i=1}^{\ell} A_{i, j+2} \phi_{j}\left(t_{0}-\tau_{i}\left(t_{0}\right)\right),
\end{array}
$$

where $K=\left[\begin{array}{ll}K_{1} & K_{2}\end{array}\right]$, according to the decomposition of the matrix $E$.

Remark 3 points out that, the compatibility of the initial condition for the closed-loop dynamics depends on the choice of the state-feedback control gain. In order to avoid this dependence we design a time-dependent state-feedback control gain $K(t)$ defined such that

$-K\left(t_{0}\right)=0$,

- $K(t)$ is continuous on $\left[t_{0}, \infty\right)$,

- $\exists \varepsilon>0$ such that $K(t)=K(\varepsilon) \triangleq K, \forall t \geq \varepsilon$.

Choosing $K\left(t_{0}\right)=0$ the condition (9) will be used to define a compatible initial condition which is independent on the state-feedback control gain. Roughly speaking equation (9) simplifies into

$$
\begin{aligned}
0=A_{03} \phi_{1}\left(t_{0}\right)+ & A_{04} \phi_{2}\left(t_{0}\right) \\
& +\sum_{j=1}^{2} \sum_{i=1}^{\ell} A_{i, j+2} \phi_{j}\left(t_{0}-\tau_{i}\left(t_{0}\right)\right) .
\end{aligned}
$$

The continuity of $K(t)$ is required to ensure the continuity of the closed-loop system trajectory. The value of $K$ will be designed in the next section in order to guarantee some performance guarantees. The parameter $\varepsilon$ can be chosen arbitrarily small in order to reduce the interval on which the controller does not provide any stability or performance guarantee. Nevertheles a compromise should be reached because a small value of $\varepsilon$ may induce important overshoots. Such an approach is natural in practice, because the operator should apply progressively the control associated with a permanent behavior, during a transient period.

\section{CONTROL SYNTHESIS}

This section is devoted to design a control law that solves Problem 1. The main tool is a Lyapunov-Krasovskii functional, that is suitable for time-delayed singular system, for which the delays satisfy the constraint (5). More particularly, we will use the Lyapunov-Krasovskii functionals that are modified to cope with time-delayed singular systems [27]. The main result is gathered in Theorem 2.

Theorem 2: Let us consider the system (1) and the assumptions detailed in the previous sections. Assume that there exist symmetric positive definite matrices $0_{n}<G_{i}=$ $G_{i}^{T} \in \mathbb{R}^{n \times n}, i \in\{1, \cdots, \ell\}$, an invertible matrix $W \in \mathbb{R}^{n \times n}$ and a matrix $Y \in \mathbb{R}^{m \times n}$ such that the relation

$$
W^{T} E^{T}=E W>0_{n}
$$

and the linear matrix inequality (LMI)

$$
\left[\begin{array}{cccccc}
\Psi & W^{T} H & Y^{T} & A_{1} W & \ldots & A_{\ell} W \\
\star & -I_{n} & 0 & 0 & \cdots & 0 \\
\star & \star & -R^{-1} & 0 & & \vdots \\
\star & \star & \star & -\left(1-d_{1}\right) G_{1} & & 0 \\
\star & \star & \star & \star & \ddots & 0 \\
\star & \star & \star & \star & \star & -\left(1-d_{\ell}\right) G_{\ell}
\end{array}\right]<0
$$

are verified, with

$$
\Psi=\operatorname{He}\left(A_{0} W+B Y\right)+\sum_{i=1}^{\ell} G_{i}
$$

and $H \in \mathbb{R}^{n \times n}$ defined by $Q=H H^{T}$. Then the state feedback (8) with the feedback gain $K=Y W^{-1}$ stabilizes the system with the Lyapunov-Krasovskii functional

$$
V\left(t, x_{t}\right)=x^{T}(t) E^{T} P x(t)+\sum_{i=1}^{\ell} \int_{t-\tau_{i}(t)}^{t} x^{T}(s) S_{i} x(s) \mathrm{d} s,
$$

with $P=W^{-1}$ and $S_{i}=P^{T} G_{i} P, i \in\{1, \cdots, \ell\}$. In addition we have the upper bound

$$
\begin{aligned}
& \mathcal{J}(\phi, u) \leq V\left(t_{0}, \phi\right)=\phi^{T}\left(t_{0}\right) E^{T} P \phi\left(t_{0}\right) \\
&+\sum_{i=1}^{\ell} \int_{t_{0}-\tau_{i}\left(t_{0}\right)}^{t_{0}} \phi^{T}(s) S_{i} \phi(s) \mathrm{d} s .
\end{aligned}
$$

Remark 4: Thanks to the structure of the singular matrix $E$, the equality (14) is verified by choosing the symmetric positive definite matrix $W$ of the form

$$
W=\left[\begin{array}{cc}
W_{1} & 0_{r \times(n-r)} \\
W_{3} & W_{4}
\end{array}\right]
$$

with $W_{1}=W_{1}^{T}>0_{r}$ and without constraint on $W_{3}$ and $W_{4}$.

Proof: Let us consider that the assumptions of the Theorem 2 are verified. Applying a Schur complement to the LMI (15), it yields

$$
\left[\begin{array}{cccc}
\Psi+W^{T} Q W+Y^{T} R Y & A_{1} W & \cdots & A_{\ell} W \\
\star & -\left(1-d_{1}\right) G_{1} & & 0 \\
\vdots & & \ddots & 0 \\
\star & \cdots & & -\left(1-d_{\ell}\right) G_{\ell}
\end{array}\right]<0
$$

Thanks to the invertibility of the matrix $W$, post-multiplying and pre-multiplying the latter inequality by $\operatorname{diag}\left(W^{-1}, W^{-1}, W^{-1}\right)$ and its transpose leads to

$$
\Phi=\left[\begin{array}{cccc}
\Omega & P^{T} A_{1} & \cdots & P^{T} A_{\ell} \\
\star & -\left(1-d_{1}\right) S_{1} & & 0 \\
& & \ddots & \\
\star & \star & & -\left(1-d_{\ell}\right) S_{\ell}
\end{array}\right]<0
$$

where

$$
\Omega=\operatorname{He}\left(A_{0}^{T} P+K^{T} B^{T} P\right)+Q+K^{T} R K+\sum_{i=1}^{\ell} S_{i} .
$$


By denoting

$$
\xi(t)=\left(\begin{array}{c}
x(t) \\
x\left(t-\tau_{1}(t)\right) \\
\vdots \\
x\left(t-\tau_{\ell}(t)\right)
\end{array}\right)
$$

we have

$$
\dot{V}\left(t, x_{t}\right)+x^{T}(t) Q x(t)+u^{T}(t) R u(t) \leq \xi^{T}(t) \Phi \xi(t)<0,
$$

for all $\xi(t) \neq 0$, where the time derivative $\dot{V}\left(t, x_{t}\right)$ exists due to the assumptions on the delays and

$$
\begin{aligned}
& \dot{V}\left(t, x_{t}\right)=\frac{\mathrm{d} V\left(t, x_{t}\right)}{\mathrm{d} t} \\
& =\dot{x}^{T}(t) E^{T} P x(t)+x^{T}(t) E^{T} P \dot{x}(t)+\sum_{i=1}^{\ell} x^{T}(t) S_{i} x(t) \\
& \quad-\sum_{i=1}^{\ell}\left(1-\dot{\tau}_{i}(t)\right) x^{T}\left(t-\tau_{i}(t)\right) S_{i} x\left(t-\tau_{i}(t)\right),
\end{aligned}
$$

that is in other words, thanks to Assumption (5)

$$
\begin{aligned}
& x^{T}(t)\left[Q+K^{T} R K\right] x(t)+\dot{x}^{T}(t) E^{T} P x(t)+x^{T}(t) E^{T} P \dot{x}(t) \\
+ & \sum_{i=1}^{\ell} x^{T}(t) S_{i} x(t)-\left(1-\dot{\tau}_{i}(t)\right) x^{T}\left(t-\tau_{i}(t)\right) S_{i} x\left(t-\tau_{i}(t)\right) \\
\leq & x^{T}(t)\left[Q+K^{T} R K\right] x(t)+\dot{x}^{T}(t) E^{T} P x(t)+x^{T}(t) E^{T} P \dot{x}(t) \\
+ & \sum_{i=1}^{\ell} x^{T}(t) S_{i} x(t)-\left(1-d_{i}\right) x^{T}\left(t-\tau_{i}(t)\right) S_{i} x\left(t-\tau_{i}(t)\right)<0 .
\end{aligned}
$$

Because by the definition of the cost function,

$$
0 \leq x^{T}(t) Q x(t)+u^{T}(t) R u(t),
$$

it implies that $\frac{\mathrm{d} V\left(t, x_{t}\right)}{\mathrm{d} t}<0$, that is the global stability of the system. As a consequence

$$
\lim _{t \rightarrow+\infty} V\left(t, x_{t}\right)=0 .
$$

In addition, by integrating the inequality (23), and due to the limit (27), one obtain the upper bound (18). That concludes the proof.

Based on Theorem 2, it is possible to build an optimization problem offering a solution to Problem 1. The idea is to select among all the state feedbacks induced by Theorem 2 , the one that minimizes the upper bound in (18), that is the initial Lyapunov-Krasovskii functional taken at $t=t_{0}$ : $V\left(t_{0}, \phi\right)$.

In practice, we would like to provide an optimization problem that is independent on the initial condition $\phi$ in order to avoid a new controller synthesis for each initial condition and also the dependency of the controller on the initial condition. Following this approach, we assume that the initial condition is a stochastic realization of a zero-mean and identical and independent process, then $\mathbb{E}[\phi(t)]=0_{n \times 1}$ and $\mathbb{E}\left[\phi(t) \phi(t)^{T}\right]=I_{n}, \forall t \in\left[t_{0}-\bar{h}, t_{0}\right]$.
Under these assumptions, we consider the expectation of the upper bound $V\left(t_{0}, \phi\right)$ as the cost to miminize:

$$
\mathbb{E}\left[V\left(t_{0}, \phi(\cdot)\right)\right]=\operatorname{Tr}\left(E P+\sum_{i=1}^{\ell} \tau_{i}\left(t_{0}\right) S_{i}\right),
$$

which can be approximated by

$$
\operatorname{Tr}\left(E P+\sum_{i=1}^{\ell} h_{i} S_{i}\right),
$$

in order to be independent also of the initial time delays.

Nonetheless, the cost defined by (28) is not express straightforwardly with variables used in Theorem 2. To circumvent this difficulty, we introduce a further auxiliary upper bound, based on the following lemma.

Lemma 1: Let us introduce a symmetric and positive definite matrix $U \in \mathbb{R}^{n \times n}$ in addition of the whole matrices introduced above. The inequality, which is an LMI in the variables,

$$
\left[\begin{array}{cc:ccc}
U & {\left[\begin{array}{c}
I_{r} \\
0_{(n-r) \times r}
\end{array}\right]} & I_{n} & \cdots & I_{n} \\
\star & W_{1} & 0_{r \times n} & \ddots & 0_{r \times n} \\
\hdashline \star & \star & & & \mathbb{D}
\end{array}\right]>0,
$$

with $\mathbb{D}=\operatorname{diag}\left(W+W^{T}-h_{1} G_{1}, \cdots, W+W^{T}-h_{\ell} G_{\ell}\right)$, implies

$$
E P+\sum_{i=1}^{\ell} h_{i} S_{i}<U
$$

Proof: Assume that the LMI (30) is feasible, then $G_{i}$ are invertible. Thanks to the relation ( $W-$ $\left.h_{i} G_{i}\right)\left(h_{i} G_{i}\right)^{-1}\left(W-h_{i} G_{i}\right)^{T} \geq 0$, it yields

$$
\left[\begin{array}{cc:ccc}
U & {\left[\begin{array}{c}
I_{r} \\
0_{(n-r) \times r}
\end{array}\right]} & I_{n} & \cdots & I_{n} \\
\star & W_{1} & 0_{r \times n} & \ddots & 0_{r \times n} \\
\hdashline \star & \star & & \multicolumn{1}{|c}{}
\end{array}\right]>0,
$$

with $\tilde{\mathbb{D}}=\operatorname{diag}\left(W\left(h_{1} G_{1}\right)^{-1} W^{T}, \cdots, W\left(h_{\ell} G_{\ell}\right)^{-1} W^{T}\right)$. Applying a Schur complement to the latter inequality results in

$$
\begin{aligned}
U & >\left[\begin{array}{cc}
W_{1}^{-1} & 0 \\
0 & 0
\end{array}\right]+W^{-T}\left(\sum_{i=1}^{\ell} h_{i} G_{i}\right) W^{-1}, \\
& =E P+\sum_{i=1}^{\ell} h_{i} S_{i}
\end{aligned}
$$

and concludes the proof.

To sum up, the optimization problem solving Problem 1 writes

Optimization problem 1:

$$
\min _{U, W, G_{1}, \cdots, G_{\ell}, Y} \operatorname{Tr}(U)
$$

over the constraints (14), (15) and (30).

The following section focuses on numerical examples to illustrate the main result. 


\section{ILLUSTRATIVE NUMERICAL EXAMPLE}

In order to illustrate the above result, a numerical example is proposed. Consider the following time delayed descriptor system where $n=2$ and $r=1$, with $\ell=2$ time varying delays, described by its matrices

$$
\begin{gathered}
E=\left[\begin{array}{ll}
1 & 0 \\
0 & 0
\end{array}\right], A_{0}=\left[\begin{array}{cc}
2 & -2 \\
-0.7 & -6.4
\end{array}\right] \\
A_{1}=\left[\begin{array}{cc}
-0.5 & 0.3 \\
0 & -0.9
\end{array}\right], A_{2}=\left[\begin{array}{cc}
-0.4 & 0 \\
1 & -0.6
\end{array}\right], B=\left[\begin{array}{l}
2 \\
1
\end{array}\right]
\end{gathered}
$$

Note that the matrix $A_{0}$ is not stable. We set $t_{0}=0$. The time varying delays are given by the time functions

$\tau_{1}(t)=0.5+0.3 \sin (t), \tau_{2}(t)=1+0.5 \sin (0.4 t), \forall t \geq 0$.

The following values are chosen for the present example: $d_{1}=0.3, d_{2}=0.2, h_{1}=0.8$, and $\bar{h}=h_{2}=1.5$. The plot of these continuous delays are given in Figure 1 below.

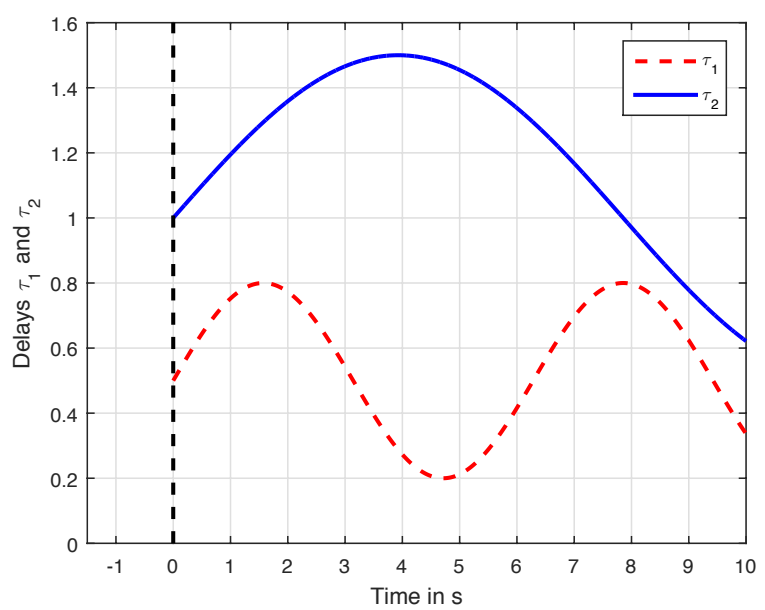

Fig. 1. System time delays $\tau_{1}(t)$ and $\tau_{2}(t)$ in function of time $t$.

Let us choose a compatible initial condition $\phi(\cdot)$, that verifies the relation (13). We select the function $\phi(\cdot)$ in the class of time affine functions of the form:

$$
\phi(t)=\left[\begin{array}{l}
\beta_{1} t+\alpha_{1} \\
\beta_{2} t+\alpha_{2}
\end{array}\right] .
$$

Precisely, we fix here $\alpha_{1}=2.5, \alpha_{2}=0.2, \beta_{1}=1.3$ and by verifying the relation (13), we obtain $\beta_{2}=2.03$.

The weighting matrices associated to the performance cost function $\mathcal{J}$ are given by the following values

$$
Q=\left[\begin{array}{cc}
10 & 0 \\
0 & 1
\end{array}\right], \quad R=1
$$

The controller is obtained by solving the constrained optimization problem 1 given in section IV. The numerical solutions are

$$
\begin{gathered}
W=\left[\begin{array}{cc}
0.192 & 0 \\
0.169 & 1.220
\end{array}\right] ; P=W^{-1}=\left[\begin{array}{cc}
5.201 & 0 \\
-0.720 & 0.820
\end{array}\right] ; \\
G_{1}=\left[\begin{array}{cc}
0.005 & 0.0704 \\
0.070 & 1.764
\end{array}\right] ; G_{2}=\left[\begin{array}{cc}
0.034 & -0.099 \\
-0.099 & 0.333
\end{array}\right] ;
\end{gathered}
$$

$$
U=\left[\begin{array}{cc}
11.473 & -0.878 \\
-0.8778 & 1.615
\end{array}\right] ; Y=\left[\begin{array}{ll}
-2.000 & -1.000
\end{array}\right]
$$

The state feedback gain is thus given by the relation $K=$ $Y P$ yielding the numerical value:

$$
K=\left[\begin{array}{ll}
-9.682 & -0.820
\end{array}\right] \text {. }
$$

Notice first that it results in the costs $\operatorname{Tr}(U)=13.08$ and $\operatorname{Tr}\left(E P+\sum_{1}^{2} h_{i} S_{i}\right)=10.49$. The gap between these two costs comes from the linearization of the nonlinear inequality (32) in Lemma 1.

In order to impose the continuity of the trajectories in closed loop, a modification of the control input as mentioned at the end of section III is applied. The control is given by

$$
u(t)=K(t) x(t)=\alpha(t) K x(t),
$$

where the weight $\alpha(\cdot)$ is defined as, with $\varepsilon=0.5$,

$$
\alpha(t)=\left\{\begin{array}{l}
\frac{1-e^{-500 t^{2}}}{1-e^{-500 \varepsilon^{2}}}, \text { if } t \in[0, \varepsilon], \\
1, \text { if } t \geq \varepsilon .
\end{array}\right.
$$

The weight $\alpha(\cdot)$ is depicted on Figure 2 and should be viewed as a bumpless control concerning the continuity at the origin.

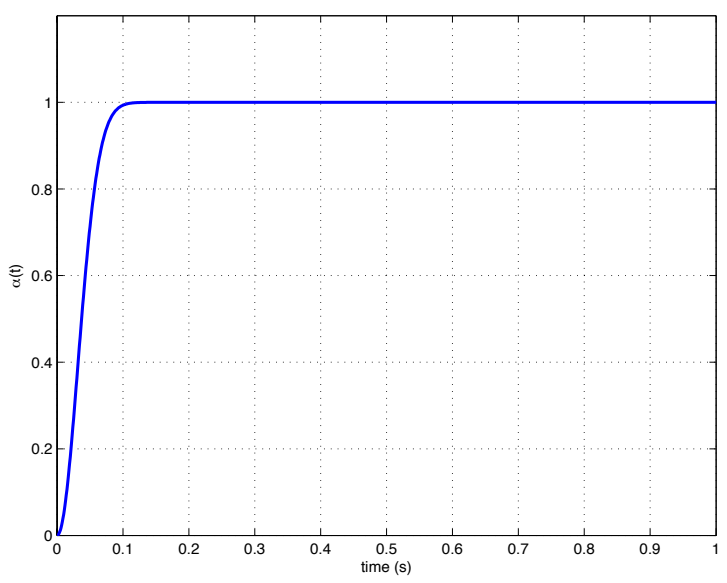

Fig. 2. Additional weight $\alpha(t)$ in function of time $t$.

Figure 3 shows the trajectory of the system. We can see that the state trajectory converges to the origin. A zoom on the time axis in Figure 4 allows to compare the control law $u(t)=\alpha(t) K x(t)$ and the pure state feedback $K x(t)$. It is emphasized that the discontinuity is replaced thanks to $\alpha(\cdot)$ by a smooth peak.

Now in order to emphasize how the operator may influence the behavior of the trajectories $x(t)$ and the controlled input $u(t)$, the same optimization problem is considered by modifying only the weights of the cost $\mathcal{J}$, all other parameters being the same:

$$
Q=\left[\begin{array}{cc}
10 & 0 \\
0 & 1
\end{array}\right], \quad R=0.01
$$



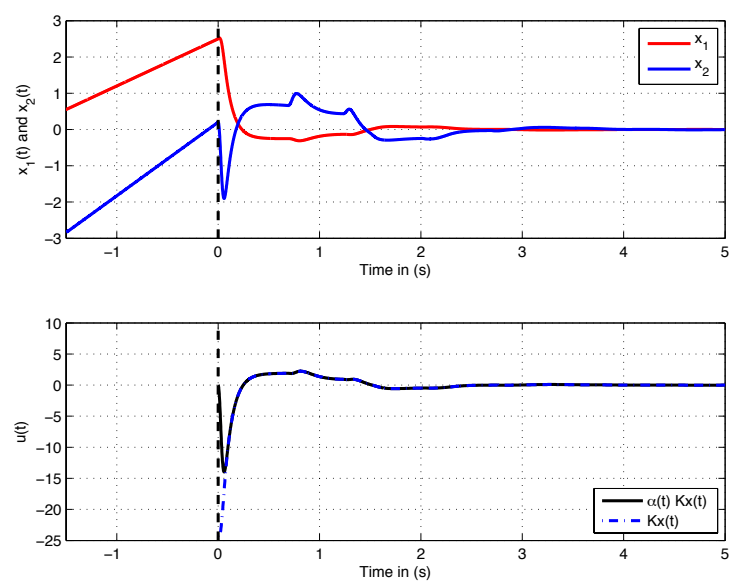

Fig. 3. State trajectories $x_{1}(t), x_{2}(t)$ and the control input $u(t)$ in function of time $t$.
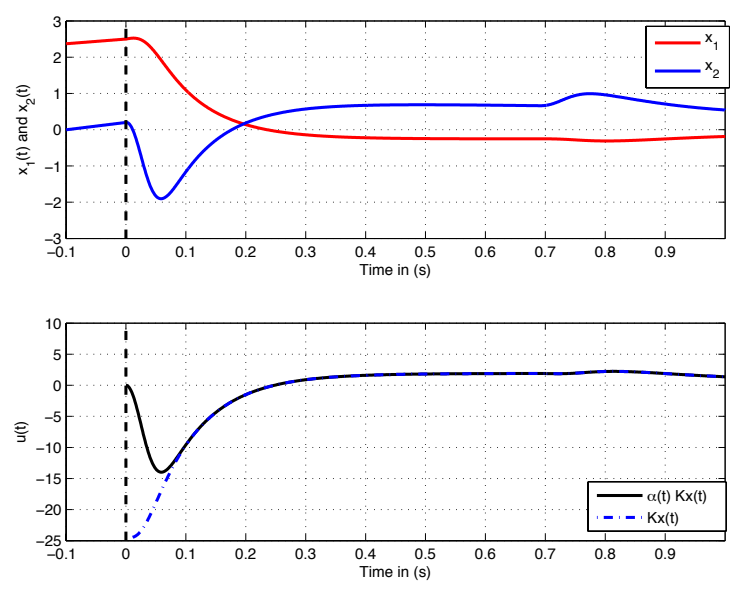

Fig. 4. Zoom on state trajectories $x_{1}(t), x_{2}(t)$ and the control input $u(t)$ in function of time $t$. The control input $u(t)=\alpha(t) K x(t)$ is compared to the time invariant feedback $K x(t)$.

which leads to the state feedback

$$
K=\left[\begin{array}{ll}
-8.415 e 2 & -0.4984 e 2
\end{array}\right] \text {. }
$$

The weight $R$ of the controlled input being reduced with respect to the initial illustration, the state feedback gains increase consequently. The time trajectories are depicted on Figure 5 . The component $x_{1}(t)$ tends faster to the origin, and a more significative peak appears for the component $x_{2}(t)$ and the control $u(t)$. This modification can be also shown on the phase portrait on Figure 6. This academic illustration points out the crucial important of the weights tuning for the

\section{CONCLUSION}

Motivated by a real problem in cold rolling of steel we proposed a guaranteed cost control design for descriptor systems with time-varying delays. We first analyzed the
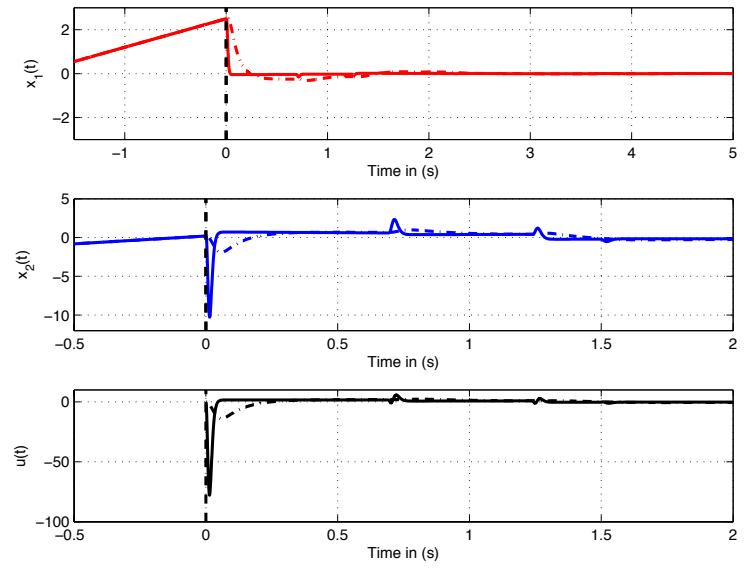

Fig. 5. Comparison between the time trajectories induced by two distinct sets of weights in the cost $\mathcal{J}$.

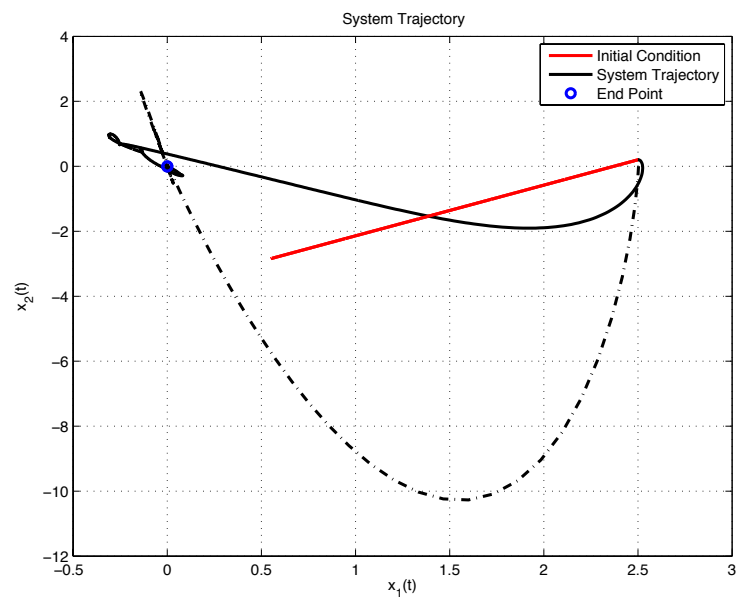

Fig. 6. Comparison between the trajectories induced by two distinct sets of weights in the cost $\mathcal{J}$. The line consists in the initial simulation and the dot dashed one to the second one. The red part of the trajectory corresponds to the system history traced for $t \in[-\bar{h}, 0]$.

existence and uniqueness of the solution for the class of systems under study. Next, we proposed a control design based on the minimization of a cost function expressing an overall energy of the system. The main tool utilized for this design was a modified Lyapunov-Krasovskii functional that takes into account the singular nature of the system. It is important to emphasize that our solution can be easily implemented since the controller design is expressed in LMIs form. Numerical simulations illustrates the effectiveness of the proposed procedure.

\section{REFERENCES}

[1] S. L. Niculescu, Delay Effects on Stability: A Robust Control Approach. Springer-Verlag, New York, 2001.

[2] J. Richard, "Time-delay systems: an overview of some recent advances and open problems," Automatica, vol. 39, pp. 1667-1694, 2003. 
[3] I.-C. Morărescu, S.-I. Niculescu, and K. Gu, "Stability crossing curves of shifted gamma-distributed delay systems," SIAM Journal on Applied Dynamical Systems, vol. 6, no. 2, pp. 475-493, 2007.

[4] I.-C. Morărescu, W.Michiels, and M. Jungers, "Effect of a distributed delay on relative stability of diffusely coupled systems, with application to synchronized equilibria," International Journal of Robust and Nonlinear Control, vol. 26, no. 7, pp. 1565-1582, 2016.

[5] W. Michiels, V. Van Assche, and S.-I. Niculescu, "Stabilization of time-delay systems with a controlled, time-varying delay and applications," IEEE Transactions on Automatic Control, vol. 50, no. 4, pp. 493-504, 2005.

[6] W. Michiels and S.-I. Niculescu, Stability and Stabilization of Time-Delay Systems: An Eigenvalue Based Approach, ser. Advances in Design and Control 12. SIAM Publications, Philadelphia, 2007.

[7] A. Seuret and F. Gouaisbaut, "Wirtinger-based integral inequality : Application to time-delay systems," Automatica, vol. 49, no. 9, pp. 2860-2866, 2013

[8] H. G. X. Li and K. Gu, "Delay-independent stability analysis of linear time-delay systems based on frequency discretization," Automatica, vol. 70, pp. 288-294, 2016.

[9] S. Campbell, Singular Systems of differential equations. Pitman Publishing Limited, London UK, 1980.

[10] L. Dai, Singular control systems. Springer, Berlin, 1989.

[11] W. L. Roberts, Cold Rolling of Steel, ser. Manufacturing Engineering and Materials Processing. Dekker, New York, USA, 1978.

[12] M. Asghar, M. Jungers, I.-C. Morarescu, A. Khelassi, and J. Francken, "Tandem cold rolling mill modeling for multi-variable control synthesis," in 17th IFAC Symposium on Control, Optimization and Automation in Mining, Mineral and Metal Processing, Vienna, Austria, 2016, p. Industrial paper, https://hal.archives-ouvertes.fr/hal-01393445.

[13] E. Orowan, "The calculation of roll pressure in hot and cold flat rolling," in Proceedings of the Institution of Mechanical Engineers, vol. 150, 1943, pp. 140-167.

[14] D. R. Bland and H. Ford, "The calculation of roll force and torque in cold strip rolling with tensions," in Proceedings of the Institution of Mechanical Engineers, vol. 159, June 1948, pp. 144-153.
[15] — "An approximate treatement of the elastic compression of the strip in cold rolling," Journal of iron and Steel Institute, vol. 171, pp. 245-249, July 1952.

[16] G. F. Bryant, The Automation of Tandem Mills. The British Iron and Steel Institute, UK, 1973.

[17] J. Pittner and M. A. Simaan, Tandem Cold Metal Rolling Mill Control. Springer, London UK, 2011.

[18] M. Jelali, "Performance assessment of control systems in rolling mills - application to strip thickness and flatness control," Journal of Process Control, vol. 17, pp. 805-816, 2007.

[19] M. Laugier, M. Tornicelli, C. Silvy-Leligois, D. Bouquegneau, D. Launet, and J. A. Alvarez, "Flexible lubrication concept, the future of cold rolling lubrication," in Proceedings of the Institution of Mechanical Engineers, Part J: Journal of Engineering Tribology, vol. 225 , no. 9,2011 , pp. $949-958$.

[20] M. Laugier, M. Tornicelli, J. Cebey, D. L. Peris, A. Devolder, R. Guillard, and F. Kop, "Flexible lubrication for controlling friction in cold rolling, crucial to be successful for the AHSS challenge," in Proceedings of the METEC and 2nd European Steel Technology and Application Days (ESTAD), Düsseldorf, Germany, 2015.

[21] E. Fridman, "Stability of linear descriptor systems with delay: a lyapunov based approach," Journal of Mathematical Analysis and Applications, vol. 273, pp. 24-44, 2002.

[22] S. Xu, P. Dooren, R. Stefan, and J. Lam, "Robust stability and stabilization for singular systems with state delay and parameter uncertainty," IEEE Transactions on Automatic Control, vol. 47, pp 1122-1128, 2002

[23] E. B. A. Haidar, "Exponential stability of singular systems with multiple time-varying delays," Automatica, vol. 45, pp. 539-545, 2009.

[24] Z.-G. Wu, J. Park, H. Su, and J. Chu, "Reliable passive control for singular systems with time-varying delays," Journal of Process Control, vol. 23, pp. 1217-1228, 2013.

[25] S. Xu and J. Lam, Robust control and filtering of singular systems. Springer-Verlag, Germany, 2006.

[26] J. Hale and S. Lunel, Introduction to function differential equations. Springer, New York, 1993.

[27] E. Fridman, Introduction to time delay systems. Birhkäuser, 2014. 